\title{
Implementation of Persistent Relay Carrier Sensing Multiple Access Cooperative Communication Protocol for Ad Hoc Network
}

\author{
Smruti Pore \\ Assistant Professor, Department of Electronics Engineering, DKTES’ TEI, Ichalkaranji
}

\begin{abstract}
With increasing demand of network coverage and congestion free network traffic, cooperative communication is getting more and more attention of network researchers. Cooperation communication is explored in number of ways. Such various techniques are studied and one method is discussed in this paper. PRCSMA- Persistent Relay Carrier Sense Multiple Access Protocol is very efficient protocol which gives good results for cooperative communication. This protocol is implemented using NS2 (Network Simulator). Its analysis is carried out in this paper. This will be useful to compare advantages of cooperative communication over regular wireless communication.
\end{abstract}

Keywords: Cooperative Communication, Relaying, PRCSMA Protocol, NS2 ( Network Simulator)

\section{Introduction}

Now a day's much interest has been involved in the design of wireless network for local area communication A wireless local area network (WLAN), is a network which connects two or more devices in limited area such as a school, computer labs, homes, office buildings by means of wireless communication. This gives flexibility to users to move freely within a range and still be connected to the network. Study group 802.11 under IEEE project 802 works for developing international standard for wireless local area networks.

In 802.11 WLAN carrier sense multiple access with collision avoidance (CSMA/CA) protocol is used which is a random access scheme used for medium access. DCF is one of the mechanisms in CSMA/CA used to access the medium in WLAN. This standard also explains point coordination function (PCF). It is a centralized MAC protocol that supports collision free and time bounded services.

In wireless communications, multiple-input and multiple output (MIMO) technology is quite successful to face the challenges of limited radio spectrum and to reduce channel losses. Despite, there is hardware difficulty to deploy multiple antennas on a small mobile node. Cooperative communication overcomes this disadvantage with existing infrastructure.

The idea of cooperative communication is, to utilize the broadcasting mode of wireless transmissions, some neighboring nodes may act as relay nodes to provide the data transfer from a source to a destination node. Thus, cooperation improves the communication's reliability and bandwidth efficiency, without the necessity of additional antennas at each node.

In addition, the cooperative relay schemes can be categorized into three sub categories, called amplify-and-forward (AF), decode-and-forward (DF), and compress-andforward (CF). In an AF scheme, the relay only amplifies and forwards a received noisy signal to the destination node. Here the noise level in a signal is also enlarged. In a DF scheme, the relay decodes the received signal and reencodes the signal. It is then forwarded to the destination node. It improves the signal to noise ratio. Because of simplicity, AF and DF schemes are commonly used schemes in designing distributed cooperative medium access control (MAC) protocols.

In recent studies it is observed that, cooperative communications works at the first layer that is physical layer along with layers 
two and three of the protocol stack i.e. the medium access control (MAC) and network layer. For efficient cooperation in wireless Local Area Network a MAC layer protocol is focused depending on IEEE 802.11, distributed coordination function (DCF). A source first transmits its own data by IEEE 802.11 DCF and if the transmission of the source fails a potential neighbor node retransmits the data to the destination after the backoff process. Thus cooperative communication is used for automatic repeat request (ARQ) scheme by extending that of legacy IEEE 802.11.

Cooperative ARQ scheme is used for decreasing packet transmission delay along with increased reliability through relaying, and improve the network throughput. However, the cooperative transmission has limited scope, and the overhead to the relay is also the major hurdle. The design of CMAC protocols can be divided in three processes namely 1.Cooperation decision, 2.Relay selection and 3.Implementation of relaying protocol. These should improve performance of wireless communication. Existing cooperative MAC protocols can be categorized into proactive and reactive schemes. In a proactive scheme, relay selection is done before the main data transmission between source and destination whereas in a reactive scheme relay selection is performed after source to destination data transmission fails.

\section{Persistent Relay Carrier Sensing Multiple Access Protocol}

In this paper implementation of PRCSMA MAC protocol is discussed and evaluated. PRCSMA protocol is used for a distributed Cooperative ARQ (Automatic Retransmission Request) Scheme in IEEE 802.11 wireless network to enhance its performance parameters and extend coverage.

PRCSMA Cooperative Communication Protocol is implemented in Network Simulator 2.35. Here Ad-hoc On-Demand Distance Vector (AODV) Routing is used for AdHoc network with wireless channel which consists of omni-directional antenna for all nodes. The area of dimension of topography is considered to be $1500 \mathrm{~m} \times 1500 \mathrm{~m}$.

\subsection{Cooperative Scenario}

The focus of this system is on the design and analysis of an MAC protocol that allows executing a distributed and cooperative automatic retransmission request (ARQ) scheme in wireless networks. These schemes exploit the broadcast nature of the wireless channel in the following manner; once a destination station receives a data packet containing errors, it can request a set of retransmissions from any of the relays which overheard the original transmission. retransmissions from the relays might be attained at higher transmission rates and they may allow for the exploitation of either space or time diversity. With such a distributed scheme, it is possible to improve the channel usage as well as to extend the coverage of the transmissions. Consider the example illustrated in Figure 4.1. It represents a multi rate system, such as the IEEE 802.11 or WiMax standards, where the achievable transmission rate between any pair of source and destination stations depends, among other factors, on the signal strength at the receiver. Typically, the higher the distance between transmitter and receiver, the lower the achievable transmission rate is for a given network configuration. This allows for idealizing a scenario whereby it is possible to define different transmission rate areas surrounding any transmitting station, as illustrated in the example of Figure 4.1. The station $\mathrm{S}$ represents a source station attempting to transmit a data packet to the destination station D. There are four available transmission rates $\mathrm{R} 4>\mathrm{R} 3>\mathrm{R} 2>\mathrm{R} 1$. The station $\mathrm{D}$ lies within the $\mathrm{R} 1$ region of the station $\mathrm{S}$, and thus communication will be performed at the lowest available transmission rate. This means, in turn, that a retransmission from the station $S$ to the station D will have the highest possible cost in terms of channel time use. However, if the station $\mathrm{D}$ requests different retransmissions from the set of relay stations 
r1, ..., r4, with whom communications might be performed at higher data transmission rates, for example, at $\mathrm{R} 4$, then, the total time required for the complete transmission process may be reduced, and thus, the channel usage increased. Although it would be desirable to be able to tailor near optimum protocols to get the most of the cooperative-prone nature of wireless communications, technological evolution is somehow constrained by economical drivers and the so called imperative backwards compatibility.

It is not possible to deploy completely novel devices as technology moves forward, and thus, it is of utmost interest to develop novel proposals that can at least coexist with currently available technology. With this idea in mind, a novel MAC protocol that can obtain the benefits of a distributed cooperative ARQ scheme while still using widely deployed commercial devices for wireless local area networks (WLANs) based on the standard IEEE 802.11 for WLANs is considered. The new proposal is called persistent relay carrier sensing multiple access (PRCSMA) protocol, and it is based on the seminal idea outlined. In addition, the protocol is analytically modeled and a performance discussion of the protocol is also presented.

\subsection{System Architecture Design}

We have proposed a Genetically Derived Secure Data Aggregation in WLAN. Initially, the CHs are chosen based on the node connectivity, which acts as a data aggregator (DAG). Then, the clustering process is executed using the genetic algorithm. When the cluster member wants to transmit the data to the aggregator, a data encryption technique are utilized. The crypto module utilized offers confidentiality to the data packet (DP), thus ensuring the authenticity and integrity of the sensed data.

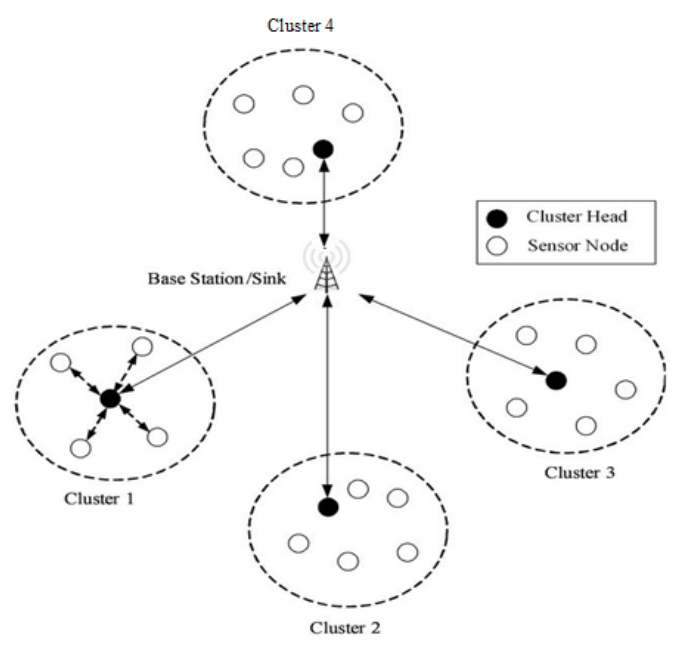

Figure 1: Cluster Formation

\subsubsection{Cluster Formation}

Clustering is a process that divides the network into interconnected sub-structures called clusters whereas Cluster is a group of nodes. In proposed system four clusters are formed, where each cluster consists of seven nodes.

In Cluster mainly there are two types a. Static Cluster and b. Dynamic Cluster. Static Cluster:- Cluster will be formed static when all nodes position will be fixed. Dynamic Cluster:- In dynamic cluster nodes are selects randomly.

\subsubsection{Cluster Head Selection :}

Upon completion of cluster creation task each node has its cluster member NID, Node Location (NL), NTE and Sink Location (SL), battery power information in its memory.

Cluster Formation takes place using two mechanisms -

1. Identifier based Clustering:- A node elects itself as the cluster head if it has the lowest/ highest ID in its neighbourhood. (Lowest-Id Heuristic)

2. Connectivity based Clustering:- The node, which has the most neighbour nodes, is elected as the cluster head.(Highest Degree Heuristic)

The node having maximum energy will calculate minimum distance of each 
node within range known as Cluster Head $(\mathrm{CH})$ and broadcast CHID to other network nodes. It also measures the node having minimum distance from the sink called Cluster Head Transmission (CHT) and broadcast CHTID to $\mathrm{CH}$. This node (CHT) will be used to transmit data toward sink if remaining $\mathrm{CH}$ energy is not sufficient for data transformation after measurement. Once each node knows it's $\mathrm{CH}$ it transmits data to $\mathrm{CH}$. Cluster head transmit processed information to the sink, this communication is single hop communication, means it make direct communication with sink.

\subsubsection{Cooperation Request}

When Erroneous reception occurs at receiver, destination station begins cooperation phase by broadcasting a message called Claim for Cooperation (CFC). Regular data transmissions in IEEE 802.11 are done after a longer silence period (DIFS), and thus cooperation phases are given priority over regular data traffic.

Here after the cluster head selection, cluster head sends the data packet to the base station. After this if base station did not receive data packet then the base station broadcasts the CFC.

A dynamic cluster will be built when the target comes close to the boundaries of multiple clusters. A demanding task issue is how the system finds the scenario when the target is approaching the boundaries, especially in a fully distributed way. We use boundary nodes to solve this issue in a fully distributed way.

\subsubsection{Cooperation Process using relay selection based on PRCSMA technique}

The network is broken into clustered layers, and the data packages travel from a lower cluster head to a higher one, in turn to the BS. Though the proposed study covers energy efficient reliable routing, it is failed to represent the basic security issues in Wireless Local Area Network. The proposed system can be enhanced by using encryption algorithm. When the sensor node sense data from environment can be encrypted and the cipher text can be forwards to base station via cluster head. The base station can decrypt and retrieve the original data.

\subsubsection{PRCSMA Analytical Model}

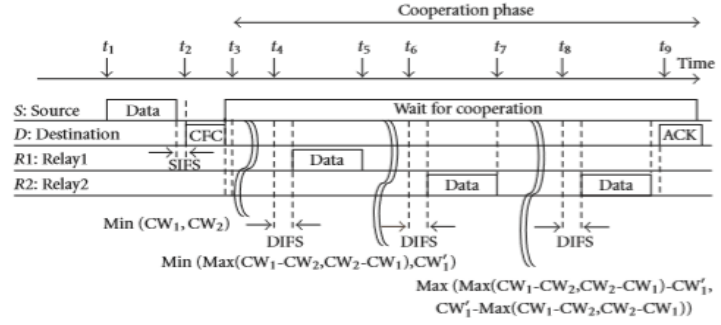

Figure 2: PRCSMA model

For the sake of understanding of PRCSMA, and before getting into the insights of the proposed analytical model, an operation of PRCSMA is presented in this section. A simple network layout with 4 stations is considered, all of them in the transmission range of each other. The basic access mode is considered, and a source station (S) transmits a data packet to destination station (D) with the support of both relays $\mathrm{R} 1$ and $\mathrm{R} 2$. The cooperation phase is represented in Fig.4.2 and explained as follows:

- At instant $\mathrm{t} 1$, station $\mathrm{S}$ sends a data packet to station D.

- Upon reception, at instant t2 station D broadcasts a CFC packet asking for cooperation to those stations in its neighbourhood (R1 and R2 in this example)

- Stations R1 and R2 receive the CFC packet and set up their backoff counters CW1 and CW2 at instant t3

- At instant $\mathrm{t4}$, the backoff counter of R1 expires (CW1), and R1 attempts a cooperative transmission.

- At instant t5, R2 resumes the backoff counter while $\mathrm{R} 1$ resets a new value for its backoff counter (CW`1) 
- At instant t6, the backoff counter of R2 expires and R2 attempts a cooperative transmission.

- At instant t7, R1 resumes the backoff counter and R2 resets a new backoff counter;

- At instant t8, the backoff counter of R2 expires and R2 attempts a cooperative transmission.

- At instant t9, station $D$ is able to properly decode the original data packet and sends back an ACK packet, indicating the end of the cooperation phase.

- All the stations then know that the cooperation phase has ended.

\section{Simulation of Protocol in NS-2}

For experimentation network simulator is used. First network scenario is created. Then clustering is done for group formation of different nodes. Finally cooperative communication is implementation among nodes.

\subsection{Network scenario}

In our simulation, each node is set with a single omni-directional antenna, and two-ray ground reflection radio propagation model are applied. Default value used for each parameter in NS-2. The carrier sensing ranges and transmission range dependent on different factors such as the environment, the transmission power and the antenna. While evaluating simulation energy consumption due to radio's energy consumption is focused. Table 4.1 gives the list of the parameters used while evaluation of simulation. To evaluate the performance of both proposed and existing grid topology scenarios is used. The performance of the scheme deliberates in terms of the packet delivery ratio and end-to-end delay that depends on the energy available in a network. On horizontal scale numbers of nodes are in use and on vertical scale transform according to performance metrics.
Table 1. List of the parameters used while designing of PRCSMA Protocol

\begin{tabular}{|c|c|}
\hline Parameter & Value \\
\hline Simulator & Ns-allionone-2.35 \\
\hline Simulation time & 25sec \\
\hline Channel type & Wireless Channel \\
\hline Propagation Model & $\begin{array}{c}\text { Propagation/Two } \\
\text { Ray Ground }\end{array}$ \\
\hline Medium & Phy/WirelessPhy \\
\hline Standard & MAC/802.11 \\
\hline Logical Link layer & LL \\
\hline Antenna & Omni Antenna \\
\hline $\begin{array}{c}\text { X dimension of the } \\
\text { topography }\end{array}$ & 1500 \\
\hline $\begin{array}{c}\text { Y dimension of the } \\
\text { topography }\end{array}$ & 1500 \\
\hline Parameter & Value \\
\hline Ad hoc Routing & AODV \\
\hline Routing & DSR \\
\hline Traffic & cbr \\
\hline
\end{tabular}

4.2 Implementation of PRSCMA Protocol 


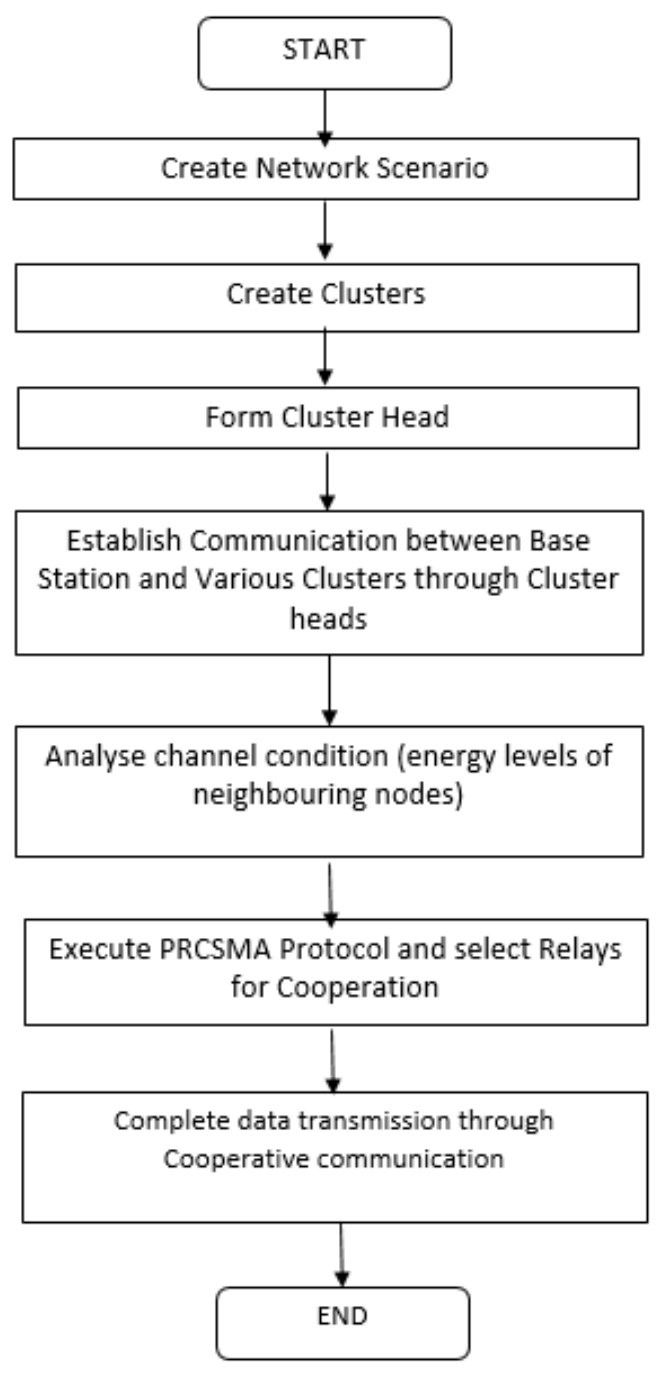

Figure 3: Flowchart of network scenario

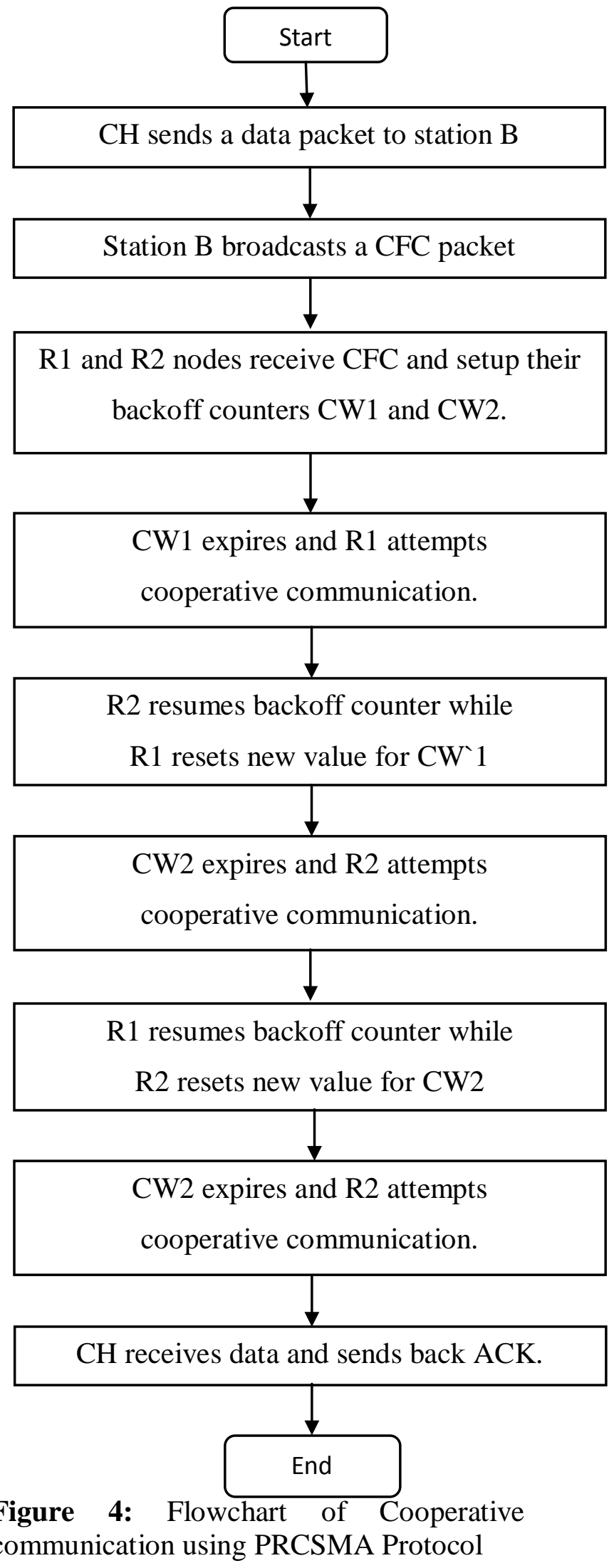


Implementation of PRCSMA Cooperative Communication Protocol is as shown in Figure 3 and Figure 4 The implementation flowchart is divided into two parts via: Overall implementation of PRCSMA Cooperative Communication Protocol and Actual PRCSMA protocol working

\subsection{Analysis Parameters for PRCSMA Protocol}

Performance parameters such as Packet Drop Rate, Throughput, Packet Delivery Ratio, are used for analysis of the implemented PRCSMA Cooperative Communication Protocol.

\subsubsection{Packet loss}

Packet loss occurs when one or more data packets sends travelling across an network fail to reach their destination. Packet loss is either caused by errors in data transmission, typically across wireless networks. Packet loss is measured as a percentage of packets lost with respect to packets sent.

$$
\begin{aligned}
& \text { Packet loss }=
\end{aligned}
$$

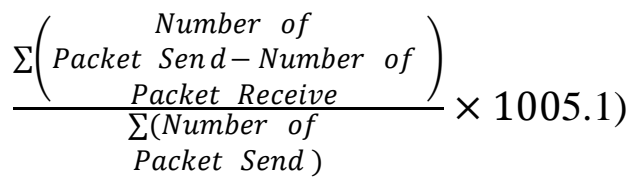

\subsubsection{Throughput}

Throughput can be defined as the ratio of the total bytes in data packets received by sink nodes to time from first packets generated at a source to last packet received by sink nodes.(Eq.5.2) The greater value of throughput states superior performance of the protocol.

$$
\text { Throughput }=\frac{\left(\begin{array}{c}
\text { Total bytes in } \\
\text { data packets } \\
\text { received by } \\
\text { sink node }
\end{array}\right)}{\begin{array}{c}
\text { Time from } \\
\text { first packet generated } \\
\text { at source to } \\
\text { last packet received } \\
\text { by sink node })
\end{array}}
$$

\subsubsection{Packet Delivery Ratio}

The packet delivery ratio (PDR) given by equation 5.3 defined as a ratio of numbers of data packets reached to target over the network to number of packets generated. The greater amount value of packet delivery ratios states superior performance of the protocol.

$$
P D R=\frac{\left.\sum \text { (Number of Packet Receive }\right)}{\sum(\text { Number of Packet Send })}(5.3)
$$

This Parameters are been evaluated and tested for different number of nodes and at different simulation time for knowing the performance of the proposed vs. existing system.

\section{Experimentation}

Experimentation is carried out in 5 parts via:

1. Cluster Formation and Cluster Head Selection.

2. Identification of weak $\mathrm{CH}$ using $\mathrm{PDR}$.

3. Initiation of CFC.

4. Relay Selection based on PRCSMA.

5. Completion of Data transmission using relaying.

\subsection{Cluster Formation and Cluster Head Selection.}

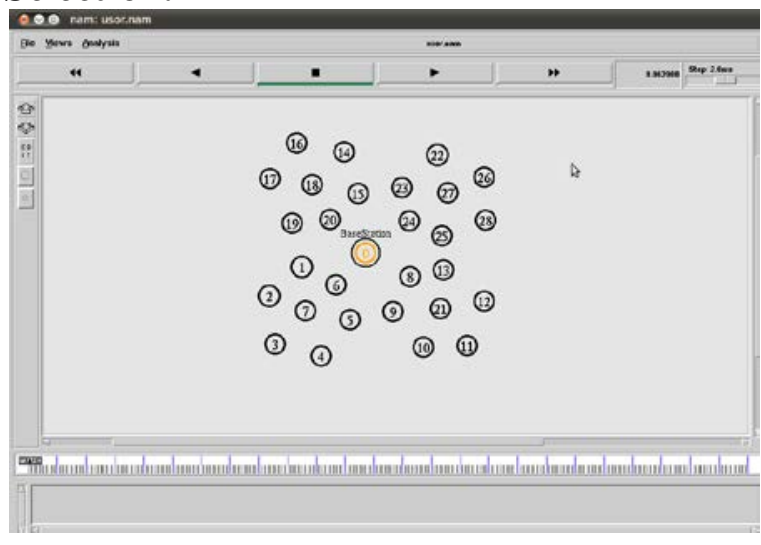

Figure 5: Simulation in NS2-Cluster Formation

shows cluster formation. Here four clusters are formed where each cluster consists of seven nodes 


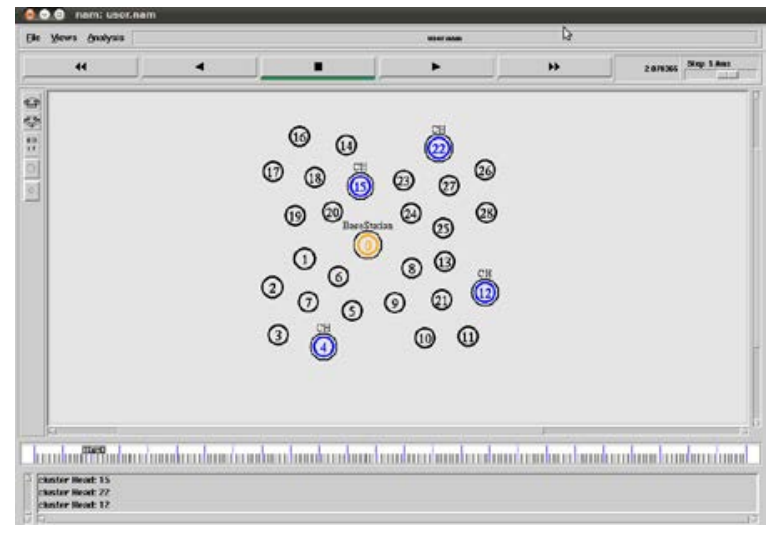

Figure 6: Simulation in NS2-Selection of Cluster Head

\subsection{Procedure to select the Cluster Head (CH) \\ Input: Cluster set with nodes. Output: $\mathrm{CH}$ selection node.}

Step 1: select all nodes as initial population. Step 2: Select evaluation set

Step 3: Apply crossover on similar power nodes.

Step 4: Apply mutation on each sensor node. Step 5: Apply fitness on all nodes power

Step 6: select best node using rout let wheel selection.

Step 7: Check GA evaluations

Step 8: Select final max energy node as $\mathrm{CH}$ node.

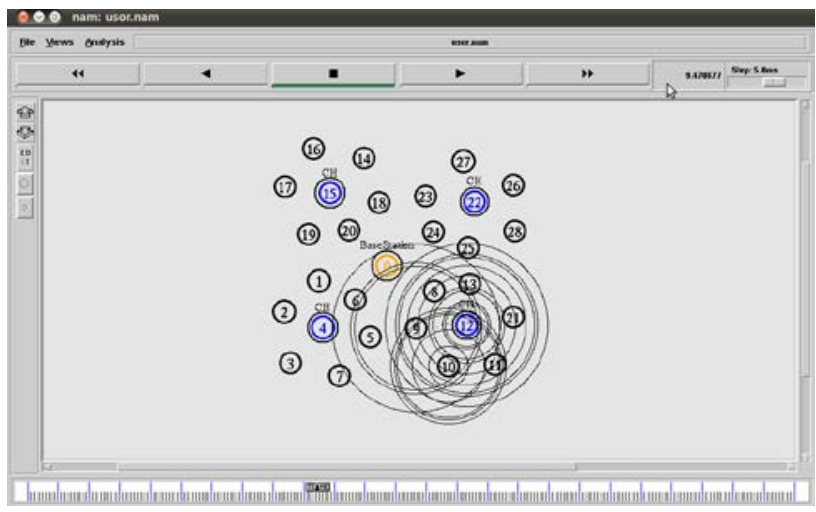

Figure 7: Simulation in NS2-Centralisation of Cluster Head

The selected Cluster Head is then forced to be positioned at the centre of the cluster, as shown in Fig. 5.4 .

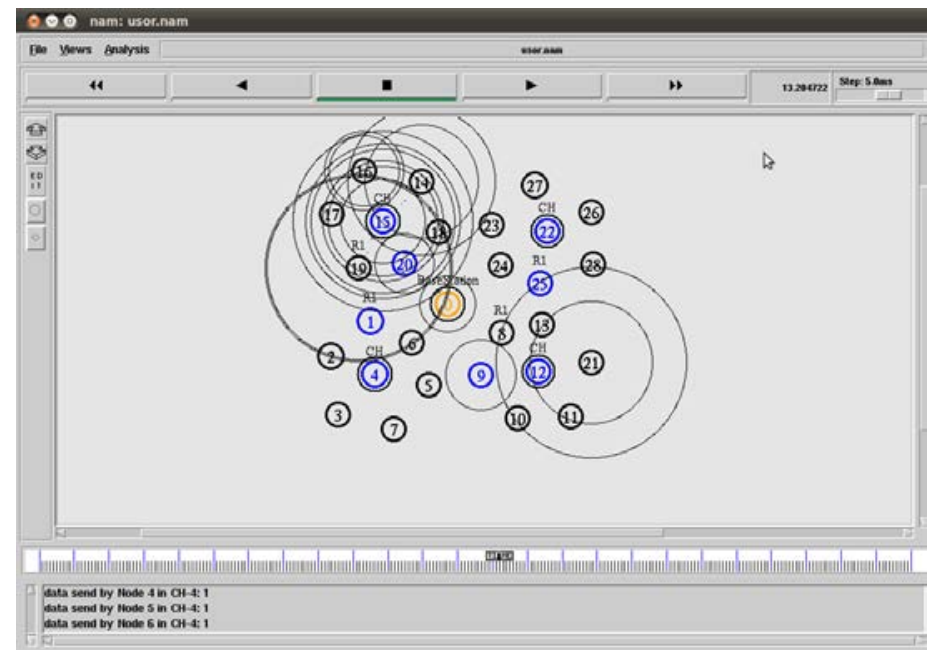

Figure 8: Simulation in NS2-Relay Selection based on PRCSMA.

\subsection{Procedure for Construction of ACO for best node selection}

Input: Initial source node $S n$, Destination node $d n$, Group of neighbor nodes nd [], each node $i d$, each node energyeng.

Output: Source to destination path when data received success.

Step 1: User first select the sn and dn Step 2: choose the packet or file $\mathrm{f}$ for data transmission.

Step 3: if (f! =null)

Step 4: read each byte b form fd when reach null

Step 5: send data, initialize cf1,cf2,pf1,pf2

Step 6: while (nd[i] when reach NULL)

$$
\begin{aligned}
& \text { Cf1 }=\text { nd[i].eng } \\
& \text { Pf1 }=\text { nd[i].id } \\
& \text { Cf2 }=\text { nsd[i+1].eng } \\
& \text { Pf2 }=\text { nd[i+1].id }
\end{aligned}
$$

Step 7: if (cf1>cf2)

Cf2=null

Pf2=null

Else

Pf1=pf2

$\mathrm{Cf} 1=\mathrm{cf} 2$;

$\mathrm{Pf} 2=$ null

Cf2=null 
Step 8: end while

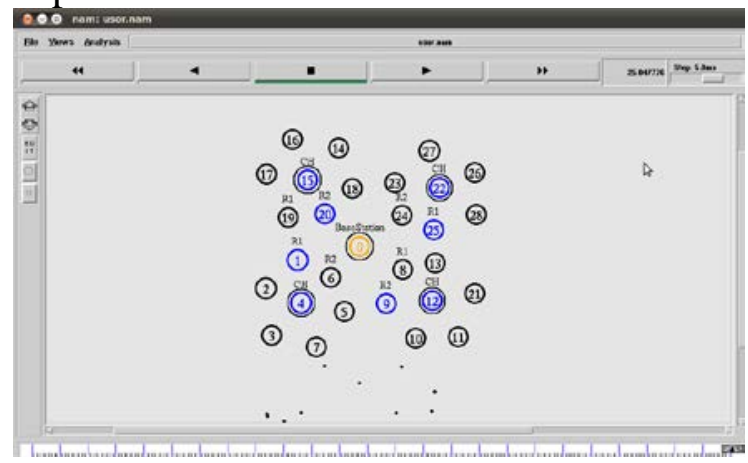

Figure 9: Completion of Data transmission using relaying.

The Figure 9 shows the completion of data transmission from $\mathrm{CH}$ to base station using cooperative nodes known as relays ( $\mathrm{R} 1$ and R2) by PRCSMA cooperative communication protocol.

\section{Results and Discussion of PRCSMA Protocol}

\subsection{Results}

Results of the experimentation on designed PRCSMA protocol are discussed further. Here two approaches are used viz; Data transmission without Cooperative communication and Data transmission with cooperative communication

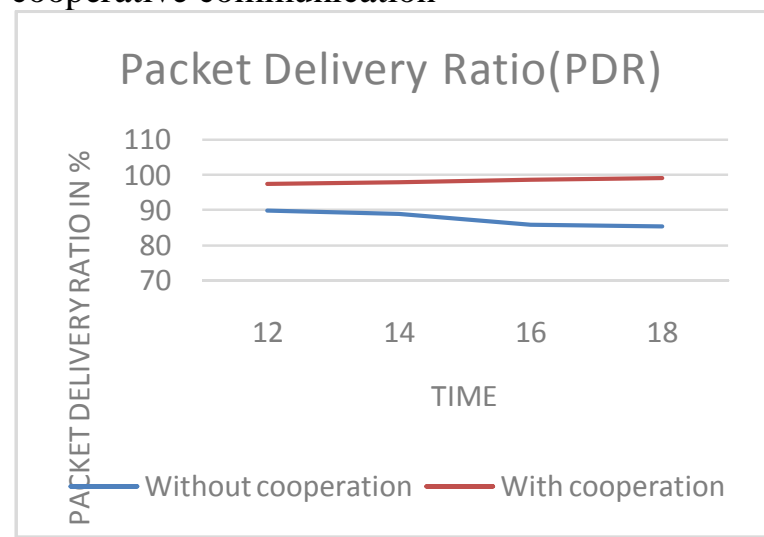

Figure 10 Plot of Packet Delivery Ratio w.r.t. various time slots

The Figure 10 shows packet delivery ratio of data transfer with cooperation remains in the range of $97 \%$ to $99 \%$, whereas packet delivery ratio of data transfer without cooperation drops from $90 \%$ to $85 \%$ as the transmission time increases.

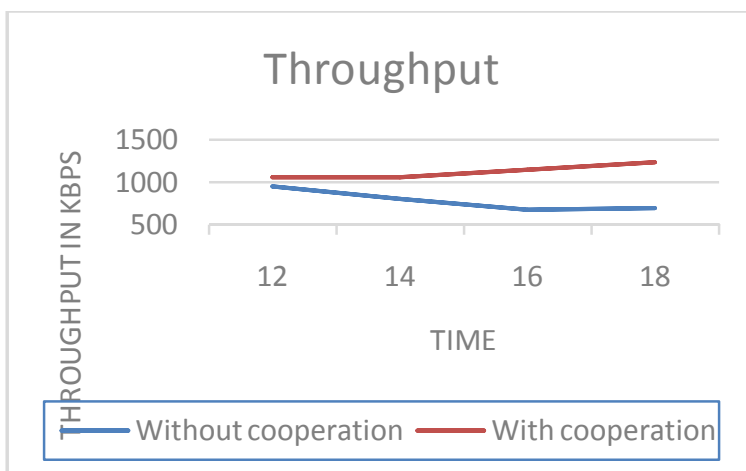

Figure 11: Plot of Throughput with respect to various time slots.

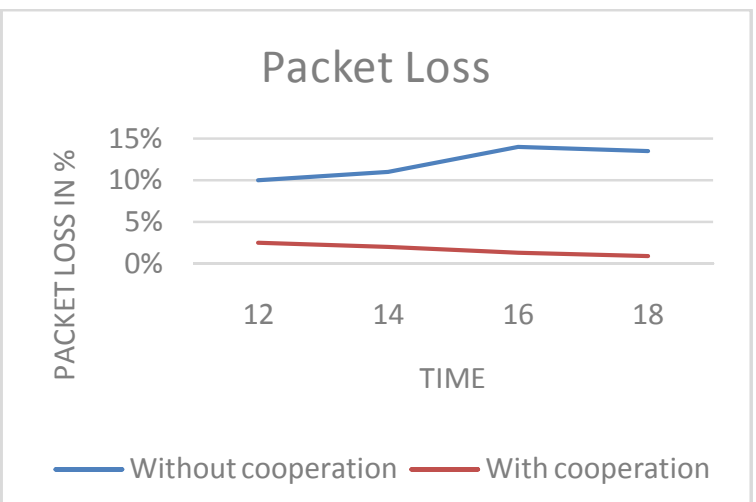

Figure 12 Plot of Packet loss with respect to various time slots.

As the time varies, the packet loss for with cooperation mode is decreased linearly and the packet loss in without cooperation mode is increased from $10 \%$ to approximately $15 \%$ with respect to time.

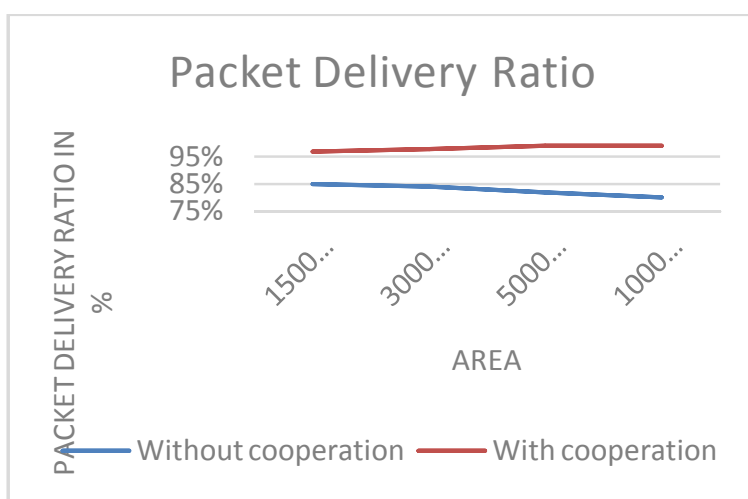

Figure 13: Plot of Packet Delivery Ratio with respect to different areas.

The Figure 13 shows packet delivery ratio without cooperation mode is 
decreased by 5\% and for with cooperation mode it ranges between $95 \%$ to $100 \%$

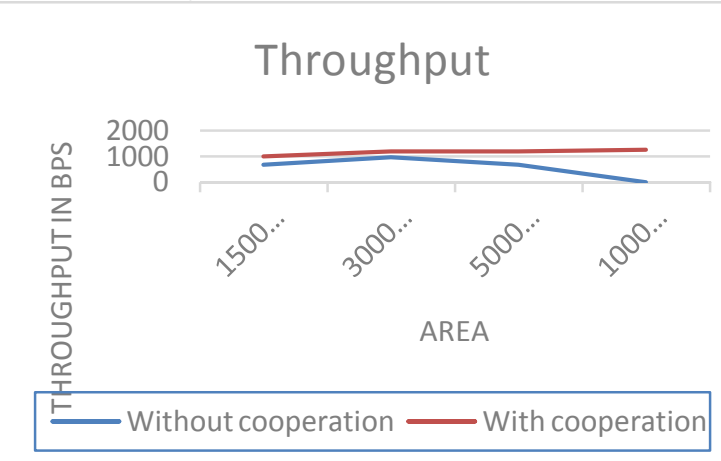

Figure 14 Plot of Throughput with respect to different areas.

The throughput of data transmission of without cooperation mode is seen to be reduced as area increased and it is reduced up to $700 \mathrm{Kbps}$ and remained constant. The throughput of with cooperation mode increases with respect to time as well as area

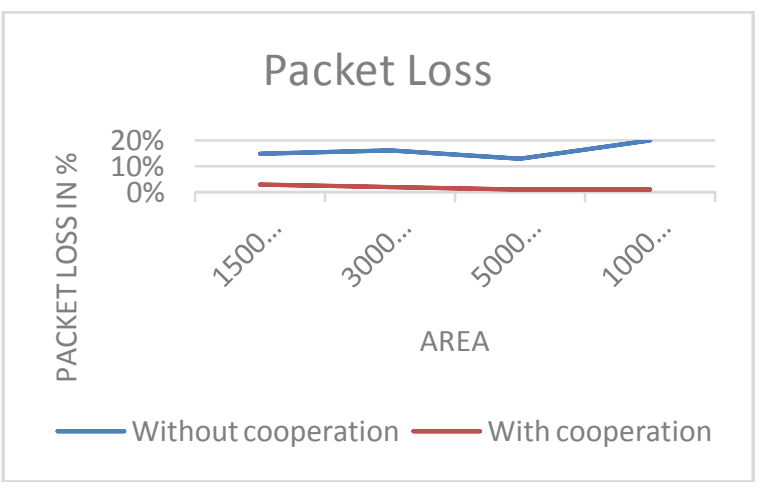

Figure 15 Plot of Packet Loss for different areas.

In Figure 15 it is seen that packet loss is lower in case of data transmission with cooperation mode as compared to that of without cooperation mode.

\subsection{Observations}

Following summary can be done based on the experiment conducted;

- Packet delivery ratio of data transfer with cooperation remains in the range of
97\% to $99 \%$, whereas packet delivery ratio of data transfer without cooperation drops from $90 \%$ to $85 \%$ as the transmission time

- It is seen that as the time varies, the packet loss for with cooperation mode is decreased linearly and the packet loss in without cooperation mode is increased from $10 \%$ to approximately $15 \%$ with respect to time.

- Packet delivery ratio without cooperation mode ranges between $80 \%$ to $85 \%$ and for with cooperation mode it ranges between $95 \%$ to $100 \%$.

- The throughput of data transmission of without cooperation mode is seen to be reduced as area increased and it is reduced up to $700 \mathrm{Kbps}$ and remained constant. The throughput of with cooperation mode increases with respect to time as well as area.

- It is seen that packet loss is lower in case of data transmission with cooperation mode as compared to that of without cooperation mode.

\section{Conclusion}

I have analyzed and carried out Persistent Relay Carrier Sensing Multiple Access cooperative communication protocol to discover benefits of cooperative communications in a distributed wireless network, we have studied two fundamental issues, namely when to cooperate and whom to cooperate with, from a PRCSMA protocol design perspective.

I have studied concept of cooperation communication with PRCSMA cooperative MAC protocol that can differentiate beneficial cooperation from unnecessary cooperation.

Effective relay selection is integrated to improve link utilization and thus increase network throughput, optimal grouping of 
helper relays for signalling overhead minimization is considered.

Simulation results demonstrate that the proposed approach with beneficial cooperation outperforms its non-cooperative counterpart in terms of throughput, Packet delivery ratio and Data Packet loss.

\section{References}

[1] C. Y. Oh and T. J. Lee, "Cooperative MAC protocol using active relays for multirate WLANs," J. Communication. Network, vol. 13, no. 5, pp. 463-471, Oct. 2011.

[2] H. Shan, H. T. Cheng, and W. Zhuang, "Cross-layer cooperative MAC protocol in distributed wireless networks,” IEEE Trans. Wireless Communication., vol. 10, no. 8, pp. 2603-2615, Aug. 2011.

[3]Z. Zhou, S. Zhou, J.-H. Cui, and S. Cui, "Energy-efficient cooperative communication based on power control and selective single-relay in wireless sensor networks," IEEE Trans. Wireless Communication., vol. 7, no. 8, pp. 30663078, Aug. 2008.

[4] X. He and F. Li, “An optimal energy efficient cooperative retransmission MAC scheme in wireless networks," in Proc. Wireless VITAE’11, Mar. 2011.

[5] Y. Liu, K. Liu, and F. Zeng, “A relaycontention-free cooperative MAC protocol for wireless networks," in Proc. IEEE CCNC'11, Jan. 2011.

[6] Sanghoon Kim and Wayne E.Stark "Simple Relay Enabled MAC (SRMAC) Protocol for Cooperative Communication" IEEE Military Communications Conference, 2013
[7] P. Liu, C. Nie, T. Korakis, E. Erkip, S. Panwar, F. Verde, and A. Scaglione, "STiCMAC: a MAC protocol for robust space-time coding in cooperative wireless LANs," IEEE Trans. Wireless Commun., vol. 11, no. 4, pp. 1358-1369, Apr. 2012.

[8] B. Schein and R. Gallager, "The Gaussian parallel relay network,” pp. 22, June 2000.

[9] J. N. Laneman, G. W. Wornell, and D. N. C. Tse, "An efficient protocol for realizing cooperative diversity in wireless networks,” IEEE Intern. Symposium Information Theory, pp. 294, June 2001.

[10] S. Geetha and C. Karpagam,” ROUTING PROTOCOLS IN WIRELESS AD-HOC NETWORK AN OVERVIEW ", International Journal of Scientific Engineering and Applied Science (IJSEAS) - Volume-2, February 2016 